Les ANNALES Les Annales de droit

DE DROIT

$11 \mid 2017$

Varia

\title{
Non-discrimination et droits des peuples
} minoritaires

Non-discrimination and rights of minority peoples

\section{Herman Blaise Ngameni}

\section{OpenEdition}

Journals

Édition électronique

URL : http://journals.openedition.org/add/541

DOI : 10.4000 /add. 541

ISSN : 2606-1988

Éditeur

Presses universitaires de Rouen et du Havre

\section{Édition imprimée}

Date de publication : 16 octobre 2017

Pagination : 131-157

ISBN : 979-10-240-0775-5

ISSN : 1955-0855

\section{Référence électronique}

Herman Blaise Ngameni, « Non-discrimination et droits des peuples minoritaires », Les Annales de droit [En ligne], 11 | 2017, mis en ligne le 16 octobre 2018, consulté le 10 décembre 2020. URL : http://

journals.openedition.org/add/541; DOI : https://doi.org/10.4000/add.541 


\title{
Non-discrimination et droits des peuples minoritaires
}

\author{
Herman Blaise NGameNI
}

Le statut de minorité est consubstantiel à la naissance des premières grandes entités politiques. Néanmoins, les ordres politiques antiques et médiévaux se caractérisaient par un certain morcellement du pouvoir institutionnel. Ainsi, au Moyen Âge, la gestion des cités ou des villes était basée sur des systèmes politico-juridiques placés sous la houlette de certains corps constitués, en l'occurrence le clergé et la noblesse qui voulaient souvent, malgré le poids des coutumes locales, imposer les mêmes règles juridiques à toutes les composantes de la population ${ }^{1}$. En Angleterre, par exemple, la Magna Carta de $1215^{2}$ ne crée pas vraiment de traitement différencié entre la majorité et la minorité au sens où l'entend Jules Deschênes c'est-à-dire «un groupe de citoyens d'un État, en minorité numérique et en position non dominante dans cet État, dotés de caractéristiques ethniques, religieuses ou linguistiques qui diffèrent de celles de la majorité de la population, solidaires les uns les autres, animés fût-ce implicitement d'une volonté collective de survie et visant à l'égalité en fait et en droit avec la majorité ${ }^{3}$ ». Une telle définition concerne manifestement les peuples minoritaires qui peuvent constituer, au moins du point de vue sociologique et/ou anthropologique, de véritables «nations» au sein d'entités étatiques qui n'ont pas encore réussi à construire une identité nationale ou politique servant de référent culturel pour l'ensemble de la population.

Néanmoins, la notion de peuple a en réalité plusieurs acceptions et peut ainsi revêtir une dimension politico-juridique (par exemple: le

1. Lire Gaetano Pentassuglia, Minorités en droit international, Strasbourg, Conseil de l'Europe, 2004, p. 25.

2. Voir notamment le $\$ 60$ de la Magna Carta qui prévoit que «toutes les coutumes et les libertés susdites que nous avons accordées doivent être observées dans notre royaume». "Nous les observerons dans nos relations avec nos hommes, tout comme elles devront être observées par les clercs et les laïcs avec leurs propres hommes. »

3. Proposal Concerning a Definition of the Term «Minority», UN Doc. E/CN.4/Sub.2/ $1985 / 31, \S 181$. 
peuple français) ou avoir une connotation sociologique et identitaire. $\mathrm{Au}$ reste, dans le concept de "peuple minoritaire ", le mot "peuple» doit être appréhendé sous l'angle de la sociologie et de l'anthropologie puisqu'il fait ici référence à un ensemble de personnes présentant les mêmes éléments d'identification du point de vue de la race, des caractéristiques ethniques, de la langue, de la religion ou de la culture et qui ayant souvent développé un fort sentiment d'appartenance à une communauté, peuvent aisément être distinguées du reste de la population d'un État.

En Europe, il semble que pendant des siècles, fut d'abord considéré comme des peuples minoritaires un ensemble de personnes professant une foi différente de celle de la majorité de la population. Dans l'Antiquité, à l'exception de quelques règnes comme celui de Néron réputé pour son intolérance religieuse, l'Empire romain était en général plutôt indulgent à l'égard des minorités religieuses. Ce libéralisme était inhérent à la structure de la société basée sur le paganisme et le polythéisme et qui tolérait toutes les religions ou tous les dieux tant que ceux-ci ne représentaient aucune menace pour le culte qui était dû à l'empereur. Plus tard, le catholicisme triomphant instaura un ordre social discriminatoire ${ }^{4}$ à l'égard des juifs, des musulmans et des hérétiques ${ }^{5}$.

L'implosion de l'unité chrétienne du monde occidental liée à la réforme luthérienne amorcée $\mathrm{au} \mathrm{Xv}^{\mathrm{e}}$ siècle et à la guerre de Trente Ans qui éclata au $\mathrm{XVI}^{\mathrm{e}}$ siècle contribua à mettre en branle une réflexion sur la situation des minorités qui étaient alors essentiellement religieuses. Cependant, les souverains de cette époque ne manifestèrent aucun enthousiasme à l'idée d'octroyer des droits spécifiques aux minorités. $\mathrm{Au}$ contraire, le traité de Westphalie (1648) conclu entre la France, l'Empire romain germanique et leurs alliés respectifs vint confirmer le présupposé selon lequel «la religion du prince est celle du pays ${ }^{6}$ » et reconnut uniquement les trois principales communautés religieuses (catholique, luthérienne, calviniste) qui pouvaient se voir octroyer certains droits civils et politiques. D’après Gaetano Pentassuglia, «les garanties accordées par le traité visaient soit des régimes complets de redistribution des territoires (pour exemple les accords passés dans le

4. Peter Kovacs fait tout de même remarquer qu'au début du Moyen Âge «appartenir à une minorité n'impliquait pas de soumission ou de statut de jure ou de facto désavantageux, mais plutôt un statut bien défini et souvent favorable» (La protection internationale des minorités aux alentours du millénaire, Paris, A. Pedone, 2005, p. 7).

5. Gaetano Pentassuglia, op. cit.

6. Maxime traduite du latin «cuius regio eius religio». 
cadre de la paix de Westphalie) soit des cessions spécifiques de territoires (par exemple le traité de Paris de 1763 entre la Grande-Bretagne et la France qui contenait des garanties pour la communauté catholique vivant dans les régions cédées par la France), de sorte que la plupart du temps leur champ d'application était confiné au territoire pertinent ${ }^{7}$ ».

Toutefois, les traités signés entre les États chrétiens et Constantinople étaient favorables à la protection des minorités chrétiennes dans l'ensemble de l'Empire ottoman.

À la fin du XVIII ${ }^{\mathrm{e}}$ siècle, si les révolutions américaine et française débouchèrent sur des déclarations garantissant $a$ priori des droits fondamentaux individuels, ceux-ci devaient profiter en grande partie aux peuples minoritaires ${ }^{8}$.

Pendant une bonne partie du $\mathrm{XIX}^{\mathrm{e}}$ siècle, et plus exactement du traité de Vienne de $1815^{9}$ au congrès de Berlin de 1878 , le processus de construction des États-nations en Europe ne favorisa pas l'émergence des droits en faveur des minorités. Il faut attendre la fin de la première guerre mondiale pour voir la Société des Nations (SDN) esquisser un système fiable de protection des minorités. La disparition des trois grands empires (austro-hongrois, prussien et ottoman) justifiait qu'il soit défini un statut pour des peuples pouvant constituer des minorités au sein de nouvelles entités étatiques. Ce nouveau système basé notamment sur la Cour permanente de justice internationale (CPJI) s'effondra définitivement en même temps que la SDN.

Après 1945, malgré les souffrances dont ont été victimes des minorités en Europe (juifs et tsiganes) pendant la seconde guerre mondiale, les questions liées aux minorités ne furent pas favorisées par la guerre froide. En effet, pendant cette période qui dura de 1946 à 1989, les responsables politiques comme d'ailleurs les juristes furent surtout obnubilés par le thème très général des droits de l'homme cela étant dû notamment à la prédominance du dogme de l'individualisme libéral. Par ailleurs, le fait que l'Allemagne hitlérienne se servit de manière abusive de la question des minorités pour troubler la paix en Europe encouragea sans doute les États à mener une politique prudente à

7. Gaetano Pentassuglia, Minorités en droit international, op. cit., p. 25.

8. Ibid., p. 26.

9. Le traité de Vienne visait avant tout à redéfinir les frontières de l'Europe suite à la disparition du premier Empire français consécutive à la chute de Napoléon Bonaparte. 
l'égard des minorités ${ }^{10}$. Pendant longtemps, même les organisations ou institutions internationales (ONU, Conseil de l'Europe...) abordèrent avec beaucoup de circonspection la question des droits des minorités ${ }^{11}$ en préférant sans doute s'appuyer sur la conception individualiste classique des droits de l'homme.

La balkanisation de l'URSS et de la Yougoslavie coïncida avec la montée des tensions ethniques en Europe et sur d'autres continents ${ }^{12}$; ce qui favorisa un regain d'intérêt pour la question des minorités tant au niveau régional que mondial. Mais il faut reconnaître qu'avant cette période, l'article 27 du pacte international sur les droits civils et politiques de 1966 faisait tout de même référence quoique timidement aux droits des minorités. De même, la convention de l'UNESCO concernant la lutte contre la discrimination dans le domaine de l'enseignement adoptée en 1960, reconnaît aux membres des minorités nationales, le droit d'exercer des activités éducatives qui leur soient propres ${ }^{13}$.

Il faut noter également qu'en 1971, le Conseil économique et social des Nations unies fit entreprendre une étude sur la discrimination dont sont victimes les peuples autochtones; ce qui déboucha sur l'important rapport publié en cinq volumes de José Martinez Cobo ${ }^{14}$ présenté au sein

10. Le protocole de Postdam de 1945 proposa par exemple de régler la question des minorités simplement par le transfert des "populations " qui pouvaient évidemment englober des peuples minoritaires.

11. Exception faite de l'article 27 du pacte international sur les droits civils et politiques de 1966 qui énonce que "[d]ans les États où il existe des minorités ethniques, religieuses ou linguistiques, les personnes appartenant à ces minorités ne peuvent être privées du droit d'avoir, en commun avec les autres membres de leur groupe, leur propre vie culturelle, de professer et de pratiquer leur propre religion, ou d'employer leur propre langue».

12. Au début des années 1990 éclatèrent des guerres interethniques, en Europe dans la région des Balkans et en Afrique dans la région des grands lacs.

13. L'article $5 \$ 1-c$ (i, ii, iii) de ladite convention dispose que les États parties reconnaissent «c. Qu'il importe de reconnaître aux membres des minorités nationales le droit d'exercer des activités éducatives qui leur soient propres, y compris la gestion d'écoles et, selon la politique de chaque État en matière d'éducation, l'emploi ou l'enseignement de leur propre langue, à condition toutefois : (i) Que ce droit ne soit pas exercé d'une manière qui empêche les membres des minorités de comprendre la culture et la langue de l'ensemble de la collectivité et de prendre part à ses activités, ou qui compromette la souveraineté nationale; (ii) Que le niveau de l'enseignement dans ces écoles ne soit pas inférieur au niveau général prescrit ou approuvé par les autorités compétentes; et (iii) Que la fréquentation de ces écoles soit facultative».

14. Voir José Martinez Cobo, Étude du problème de la discrimination à l'encontre des populations autochtones, New York, Nations Unies, 1986-1987, 5 vol. José Martinez Cobo (professeur de sociologie équatorien) fut nommé rapporteur spécial sur la discrimination contre les peuples indigènes. (E/CN.4/Sub.2/1986/7, E/CN.4/Sub.2/ 
de la sous-commission des droits de l'homme pour la prévention de la discrimination et la protection des minorités entre 1981 et $1987^{15}$.

Aussi en 1990, une disposition favorable aux droits des enfants des populations minoritaires ou autochtones fut consacrée dans la convention des Nations unies sur les droits de l'enfant, en des termes plus précis que ceux de l'article 27 du pacte international sur les droits civils et politiques ${ }^{16}$. Mais, c'est évidemment la période postérieure à la guerre froide qui est plus favorable à l'élaboration des régimes internationaux fixant les droits des minorités. Dans le cadre de l'ONU, le principal texte non conventionnel contemporain consacré à la question des personnes appartenant à des minorités nationales, ethniques, religieuses ou linguistiques est adopté par l'assemblée générale des Nations unies en $1992{ }^{17}$.

Au niveau européen, le Conseil de l'Europe et l'Organisation pour la sécurité et la coopération en Europe (OSCE) se sont beaucoup investis ces dernières décennies dans le domaine de la promotion et de la protection des minorités. La convention-cadre du Conseil de l'Europe pour la protection des minorités (1995) et la charte européenne des langues régionales ou minoritaires (1992) sont parmi les premiers textes contraignants consacrés essentiellement aux droits des minorités. Un tel regain d'intérêt pour la question des minorités, et spécialement des peuples minoritaires, fut justifié par le fait que la fin de la guerre froide coïncida avec le triomphe de l'idéologie des droits de l'homme. Dès lors, la communauté internationale pu davantage s'intéresser aux graves discriminations et leurs cohortes de souffrance dont ont été victimes

1986/7/Add.1, E/CN.4/Sub.2/1986/7/Add.2, E/CN.4/Sub.2/1986/7/Add.3, E/CN.4/ Sub.2/1986/7/Add. $4 ; 1986)$.

15. Sur le sujet lire notamment Françoise Molin, «Vers une déclaration universelle des droits des peuples autochtones », dans Henri Giordan (dir.), Les minorités en Europe. Droits linguistiques et droits de l'Homme, Paris, Kimé, 1992, p. 493-510.

16. L'article 30 de la convention relative aux droits de l'enfant adoptée à New York le 26 janvier 1990 dispose que «dans les États où il existe des minorités ethniques, religieuses ou linguistiques ou des personnes d'origine autochtone, un enfant autochtone ou appartenant à une de ces minorités ne peut être privé du droit d'avoir sa propre vie culturelle, de professer et de pratiquer sa propre religion ou d'employer sa propre langue en commun avec les autres membres de son groupe».

17. Voir la déclaration des droits des personnes appartenant à des minorités nationales ou ethniques, religieuses et linguistiques, adoptée par l'assemblée générale dans sa résolution $47 / 135$ du 18 décembre 1992. Il faut tout de même préciser qu'avant la déclaration de 1992, l'assemblée générale des Nations unies avait fait en 1981 une déclaration sur l'élimination de toutes les formes d'intolérance et de discrimination fondées sur la religion ou la conviction. Voir la résolution 36/55 de l'AG du 25 novembre 1981. 
certaines populations. En effet, le $\mathrm{xx}^{\mathrm{e}}$ siècle est bien celui du génocide des Arméniens, des juifs, des Tsiganes, ou des Tutsis; mais aussi de la discrimination raciale aux États-Unis. En outre, la discrimination à l'égard des peuples minoritaires fut sans doute l'une des causes de l'épuration ethnique en ex-Yougoslavie.

La lutte contre la discrimination à l'égard des peuples minoritaires est sans conteste un sujet de préoccupation dans nombre d'États contemporains. Au reste, le principe de non-discrimination ${ }^{18}$ fait aujourd'hui partie de la phraséologie juridique et postule " qu'un traitement égal soit réservé à des individus égaux et implique l'existence d'une norme prescrivant l'égalité de traitement ${ }^{19}$ ». La plupart des responsables politiques ainsi que les juristes s'accordent pour reconnaître que la protection des minorités est devenue un enjeu majeur pour la préservation de la stabilité dans les États et du maintien de la paix dans le monde. Bien sûr le statut des «minorités» et plus précisément des "peuples minoritaires» mérite bien une certaine clarification en ce qui concerne notamment leurs droits. Il est constant que du point de vue juridique les peuples minoritaires sont protégés contre les discriminations c'est-à-dire contre toutes les formes d'isolement ou d'exclusion dont ils pourraient être l'objet. Mais étant donné que leurs droits sont consignés dans des instruments juridiques disparates quelles sont leur nature et leur portée réelle?

En s'intéressant de plus près à la question des peuples minoritaires, il apparaît que, si ceux-ci disposent des droits qui leur sont spécifiques et qui visent à les prémunir contre tout risque de discrimination (2), en vérité ces droits tirent leur source de l'arsenal juridique consacré à la protection de tous les individus, lequel se révèle être en réalité le point de départ de la protection des peuples minoritaires (1).

18. Ce principe découle de divers instruments internationaux notamment la déclaration universelle des droits de l'homme (art. $1^{\text {er }}$ ), la charte des Nations unies (art. $1 \S 3$ ), le pacte international relatif aux droits civils et politiques (art. $2 \$ 1$ ), la convention européenne des droits de l'homme (art. 14), la convention américaine des droits de l'homme (art. $1 \$ 1$ ), la charte africaine des droits de l'homme et des peuples (art. 2), la charte des droits fondamentaux de l'Union européenne (art. 21).

19. Frédéric Sudre, Droit européen et international des droits de l'homme, Paris, PUF, 2015, p. 415. 


\section{Le point de départ de la protection des peuples minoritaires contre la discrimination}

À l'origine, les États préoccupés par le fait d'assimiler à tout prix les peuples minoritaires qu'ils abritaient n'ont pas songé à leur octroyer des droits spécifiques compte tenu de leur particularisme. C'est pourquoi les membres des minorités comme tous les autres individus devaient se contenter du bénéfice des droits individuels et collectifs réservés à tous les citoyens (1.1) ce qu'est venu confirmer le droit très particulier à la non-discrimination (1.2).

\subsection{Le principe de la reconnaissance des droits individuels et collectifs aux membres des minorités}

Les membres des peuples minoritaires en tant que citoyens d'un État jouissent pleinement de tous les droits réservés à leurs compatriotes; qu'il s'agisse des droits individuels (1.1.1) ou des droits collectifs (1.1.2).

\subsubsection{La reconnaissance des droits individuels aux membres des minorités}

Contrairement aux États multinationaux, les États nationaux prétendent s'appuyer soit sur une seule nation préexistante, soit sur un florilège de nations qu'ils ont réussi à fusionner en une seule ${ }^{20}$. Dès lors, les institutions étatiques reposent sur une conception libérale dont la conséquence sur le plan philosophique et juridique reste la garantie exclusive des droits individuels hérités du siècle des Lumières. Ces droits sont essentiellement civils et politiques, et la question est surtout de savoir quel est leur impact sur la protection des peuples minoritaires. En vérité, si en 1789 les révolutionnaires français ont par exemple proclamé la liberté d'opinion ${ }^{21}$, c'était avant tout parce que celle-ci leur permettait de s'adonner librement à la critique philosophique à laquelle ils étaient tant attachés. Mais, le fait est que cette liberté a été accueillie avec

20. Sur la différence entre les États nationaux et les États multinationaux, lire Norbert Rouland et al., Droit des minorités et des peuples autochtones, Paris, PUF, 1996, p. 291 et suiv. Voir aussi Definition and Classification of minorities, Memorandum submitted by the Secretary-General, United nations, Commission on Human Rights, Sub-Commission on Prevention of Discrimination and Protection of Minorities, New York, Lake Success, 1950, p. 7.

21. L'article 10 de la Déclaration des droits de l'homme et du citoyen du 26 août 1789 dispose que «nul ne doit être inquiété pour ses opinions, mêmes religieuses, pourvu que leur manifestation ne trouble pas l'ordre public établi par la loi ». 
soulagement notamment par la minorité protestante et la communauté juive qui subissaient très souvent les affres de la discrimination.

Très souvent, les États qui, comme la France, ont fait le choix d'une construction jacobine de la société répugnent à octroyer des droits spécifiques aux minorités qui doivent alors se fondre dans l'ensemble du corps social pour former une entité homogène. Toutefois, il faut dire que les droits individuels qui visent à garantir le bien-être de la personne débouchent fatalement sur la préservation des intérêts d'une minorité dont les membres peuvent individuellement défendre leurs droits fondamentaux reconnus par l'ordre juridique.

Ainsi, les droits de l'homme reconnus à chaque individu et bénéficiant d'une protection internationale ne sont pas nécessairement en déphasage avec les intérêts d'un peuple minoritaire à partir du moment où ses membres peuvent s'en prévaloir dans les mêmes conditions que la majorité de la population. D'ailleurs, même l'article 27 du pacte international relatif aux droits civils et politiques (PIDCP) de $1966^{22}$ fait surtout référence aux droits individuels dont sont titulaires les membres d'un groupe minoritaire ${ }^{23}$. C'est pourquoi le Comité des droits de l'homme a admis le droit à un recours individuel au profit d'une personne issue d'une minorité sur le fondement dudit article $27^{24}$.

Les droits de l'homme auxquels peuvent prétendre les individus indépendamment de leurs origines raciales, ethniques, nationales, religieuses ou sociales prennent souvent leur source dans le droit supranational ou dans le droit national. Le contenu des droits individuels est évidemment large pour tous les citoyens d'un État démocratique et couvre aussi bien les droits-libertés tels que le droit à la participation politique, le droit à la sûreté, à la liberté au sens large, à la dignité ou au respect de sa personne, que les droits-créances à l'instar du droit à la santé, au logement, et plus globalement à l'assistance sociale. Il faut préciser qu'en plus des droits considérés comme individuels par nature, les membres des minorités détiennent dans les États démocratiques, des droits considérés comme

22. Art. 27 du PIDCP : «Dans les États où il existe des minorités ethniques, religieuses ou linguistiques, les personnes appartenant à ces minorités ne peuvent être privées du droit d'avoir, en commun avec les autres membres de leur groupe, leur propre vie culturelle, de professer et de pratiquer leur propre religion, ou d'employer leur propre langue. »

23. Pour le Comité des droits de l'homme, les dispositions de l'article 27 garantissent des droits individuels qui se différencient des droits collectifs (CCPR/C/21/Rev. 1/Add. 5, avril 1994).

24. Voir Comité des droits de l'homme, décision B. Ominayak et la bande du lac Lubicon c/Canada, 26 mars 1990. 
collectifs dans la mesure où ils sont normalement exercés par un groupe de personnes.

\subsubsection{La détention des droits collectifs par les membres des peuples minoritaires}

La notion de droit collectif peut prêter à confusion, c'est pourquoi il est utile d'en déterminer les contours. En se situant du point de vue de la philosophie juridique française, les droits ne sont collectifs que dans la mesure où ils peuvent être exercés par un groupe d'individus sans que cela puisse apparaître, contrairement à la conception canadienne par exemple, comme une consécration des privilèges au profit de certains groupes spécifiques ${ }^{25}$. D’ailleurs, la doctrine juridique française préfère employer des concepts tels que «libertés relationnelles ${ }^{26}$ ", «libertés de l'expression collective ${ }^{27}$ », ou « libertés d'action collective ${ }^{28}$ », pour faire allusion aux droits collectifs. D’une manière générale, ces derniers sont, d'une part, la liberté d'association et, d'autre part, la liberté de réunion, même s'il est possible d'y intégrer la liberté de manifestation.

Dans les États où le principe d'indivisibilité du corps social s'oppose à l'octroi de droits particuliers aux minorités, celles-ci peuvent néanmoins se servir des droits collectifs comme d'une passerelle leur permettant de défendre des intérêts qui leur sont propres. De fait, les droits à la liberté de réunion et d'association, par exemple, constituent des brèches au cœur de la conception individualiste des droits de l'homme. Malgré tout, il est assez fréquent de considérer que même les droits dits collectifs ont en réalité une assise individuelle ${ }^{29}$.

Seulement, rien ne s'oppose à ce que, dans une société démocratique, les peuples minoritaires se servent par exemple du droit à la liberté d'association pour défendre des intérêts dont les seules limites résident dans la nécessité de respecter l'ordre public et les droits d'autrui. $\mathrm{Au}$ reste, la liberté collective de s'associer est consacrée par le droit

25. Pour Gaetano Pentassuglia, par exemple, les droits des minorités font partie des droits collectifs qui au demeurant seraient en opposition avec les droits individuels (Minorités en droit international, op. cit., p. 48-49).

26. Gilles Lebreton, Libertés publiques et droits de l'homme, Paris, Sirey, 2008, p. 517.

27. Jean Duffar et Jacques Robert, Droits de l'homme et libertés fondamentales, Paris, Lextenso, 2009, p. 831.

28. Jacques Fialaire et al., Libertés et droits fondamentaux, Paris, Ellipses, 2012, p. 591.

29. En ce qui concerne la liberté d'association, voir notamment Rémy Cabrillac (dir.), Libertés et droits fondamentaux, Paris, Dalloz, 2015, p. 537 et suiv. 
international et notamment l'article $22 \mathrm{du} \operatorname{PIDCP}^{30}$. De même, à l'échelle régionale, les articles $10^{31}, 11^{32}$ et $16^{33}$ respectivement de la charte africaine des droits de l'homme et des peuples, de la convention européenne des droits de l'homme et de la convention américaine relative aux droits de l'homme, garantissent le principe de la libre association pour tous les individus qui veulent se réunir pour défendre des intérêts collectifs.

Il est vrai que, comme pour tous les citoyens, la liberté d'association des membres des minorités doit être en phase avec les principes fondamentaux qui sous-tendent un État. Ainsi, dans le cadre français, par exemple, l'article 3 de la loi du $1^{\text {er }}$ juillet 1901 relative au contrat d'association dispose que «toute association fondée sur une cause ou en vue d'un objet illicite, contraire aux lois, aux bonnes mœurs, ou qui aurait pour but de porter atteinte à l'intégrité du territoire national et à la forme républicaine du gouvernement, est nulle et de nul effet». En dehors des exigences imposées par cette disposition, le droit français se montre plutôt libéral en ce qui concerne la création d'une association, puisque celle-ci peut exister même en l'absence de toute déclaration auprès de l'administration ${ }^{34}$. Dès lors, le principal critère retenu pour désigner une association reste l'accord de volonté entre des personnes

30. Art. $22 \S 1$ et 2 du PIDCP : «1. Toute personne a le droit de s'associer librement avec d'autres, y compris le droit de constituer des syndicats et d'y adhérer pour la protection de ses intérêts. 2. L'exercice de ce droit ne peut faire l'objet que des seules restrictions prévues par la loi et qui sont nécessaires dans une société démocratique, dans l'intérêt de la sécurité nationale, de la sûreté publique, de l'ordre public, ou pour protéger la santé ou la moralité publiques ou les droits et les libertés d'autrui. Le présent article n'empêche pas de soumettre à des restrictions légales l'exercice de ce droit par les membres des forces armées et de la police.»

31. Art. $10 \$ 1$ de la charte africaine des droits de l'homme et des peuples: «Toute personne a le droit de constituer librement des associations avec d'autres, sous réserve de se conformer aux règles édictées par la loi. »

32. Art. $11 \$ 1$ de la convention européenne des droits de l'homme: «Toute personne a droit à la liberté de réunion pacifique et à la liberté d'association, y compris le droit de fonder avec d'autres des syndicats et de s'affilier à des syndicats pour la défense de ses intérêts."

33. Art. $16 \$ 1$ de la convention américaine relative aux droits de l'homme: «Toute personne a le droit de s'associer librement à d'autres à des fins idéologiques, religieuses, politiques, économiques, professionnelles, sociales, culturelles, sportives ou à toute autre fin.»

34. En droit français, une association non déclarée est une organisation de facto dénuée de toute personnalité morale ou de capacité juridique, ce qui la prive par exemple de la possibilité de se constituer un patrimoine. Voir notamment Jacques Fialaire et al., op. cit., p. 594 . 
qui «mettent en commun de façon permanente leurs connaissances ou leur activité dans un but autre que de partager des bénéfices ${ }^{35}$ ".

La permanence est ce qui permet de différencier la liberté d'association de la liberté de réunion. En effet, si cette dernière peut permettre aussi aux membres des peuples minoritaires de se rassembler dans un objectif précis, le rassemblement ne peut avoir en principe qu'un caractère temporaire $^{36}$. La liberté de réunion est garantie sous réserve qu'elle ne heurte pas les principes fondamentaux reconnus dans les États démocratiques. C'est ce qui ressort d'ailleurs des normes internationales qui consacrent la liberté de réunion, qu'il s'agisse aussi bien du PIDCP ${ }^{37}$ (art. 21), de la convention européenne des droits de l'homme ${ }^{38}$ (art. 11), de la charte africaine des droits de l'homme et des peuples ${ }^{39}$ (art. 11) ou de la convention américaine relative aux droits de l'homme ${ }^{40}$ (art. 15). La liberté de réunion est considérée comme une liberté fondamentale

35. Lire l'article $1^{\text {er }}$ de la loi du $1^{\text {er }}$ juillet 1901 relative au contrat d'association.

36. En France, par exemple, en vertu de l'article 6 de la loi du 30 juin 1881 sur la liberté de réunion: «Les réunions ne peuvent être tenues sur la voie publique; elles ne peuvent se prolonger au-delà de onze heures du soir ; cependant, dans les localités où la fermeture des établissements publics a lieu plus tard, elles pourront se prolonger jusqu'à l'heure fixée pour la fermeture de ces établissements. »

37. Art. 21 du PIDCP: «Le droit de réunion pacifique est reconnu. L'exercice de ce droit ne peut faire l'objet que des seules restrictions imposées conformément à la loi et qui sont nécessaires dans une société démocratique, dans l'intérêt de la sécurité nationale, de la sûreté publique, de l'ordre public ou pour protéger la santé ou la moralité publiques, ou les droits et les libertés d'autrui.»

38. Si le $\$ 1$ de l'article 11 de la convention européenne des droits de l'homme garantit bien la liberté de réunion pacifique et d'association, le $\$ 2$ du même article prévoit que l'exercice des droits de réunion ou d'association «ne peut faire l'objet d'autres restrictions que celles qui, prévues par la loi, constituent des mesures nécessaires, dans une société démocratique, à la sécurité nationale, à la sûreté publique, à la défense de l'ordre et à la prévention du crime, à la protection de la santé ou de la morale, ou à la protection des droits et libertés d'autrui». "Le présent article n'interdit pas que des restrictions légitimes soient imposées à l'exercice de ces droits par les membres des forces armées, de la police ou de l'administration de l'État. »

39. Art. 11 de la charte africaine des droits de l'homme et des peuples : « Toute personne a le droit de se réunir librement avec d'autres. Ce droit s'exerce sous la seule réserve des restrictions nécessaires édictées par les lois et règlements, notamment dans l'intérêt de la sécurité nationale, de la sûreté d'autrui, de la santé, de la morale ou des droits et libertés des personnes. »

40. Art. 15 de la convention américaine relative aux droits de l'homme: «Le droit de réunion pacifique et sans armes est reconnu. L'exercice de ce droit ne peut faire l'objet que des seules restrictions qui, prévues par la loi sont nécessaires dans une société démocratique dans l'intérêt de la sécurité nationale, de la sûreté et de l'ordre public ou pour protéger la santé ou la moralité publiques, ou les droits ou les libertés d'autrui.» 
dans les sociétés démocratiques ${ }^{41}$, à l'instar d'ailleurs de la liberté de manifestation qui « est un moyen pour un groupe de personnes utilisant la voie publique, d'exprimer une revendication ou une protestation à l'égard des pouvoirs publics ${ }^{42}$ ".

S'il est possible de croire que dans une démocratie les membres des peuples minoritaires puissent se prévaloir non seulement des droits individuels, mais aussi des droits d'action collective, c'est pour la simple raison qu'ils bénéficient des effets du droit à la non-discrimination.

\subsection{Le bénéfice par les minorités du droit à la non-discrimination}

Le bénéfice du droit à la non-discrimination par les minorités est le corollaire du principe d'égalité devant la loi pour tous les citoyens. Il revêt une certaine importance du point de vue juridique (1.2.1) d'où l'intérêt d'en mesurer la portée (1.2.2).

\subsubsection{L'importance du droit à la non-discrimination pour les minorités}

Le droit à la non-discrimination est un avatar du principe d'égalité entre les sujets de droit dont l'article premier de la Déclaration des droits de l'homme et du citoyen de 1789 (DDHC) est l'une des plus grandes illustrations ${ }^{43}$. Il repose sur le postulat selon lequel tous les êtres humains sans exception ont droit à une égale dignité censée les mettre à l'abri de tout traitement juridique différencié qui pourrait remettre en cause leurs droits fondamentaux. Ainsi, « toute personne quelle que soit son origine familiale, culturelle, raciale, ethnique, religieuse [...] doit être reconnue comme une fin en soi; comme représentant l'humanité ${ }^{44}$ ".

Au fil du temps, le droit à la non-discrimination s'est imposé comme une nécessité visant à protéger notamment les peuples minoritaires contre des agissements qui ont pu, comme ce fut le cas notamment pour les Arméniens, les juifs ou les Tsiganes pendant la première moitié $\mathrm{du} \mathrm{xx}^{\mathrm{e}}$ siècle, aller jusqu'à menacer leur existence. Mais il va sans dire

41. Dans le cadre français, il n'y a plus aucun doute sur la fondamentalité de la liberté de réunion au sens de la loi du 30 juin 2000 qui a instauré le référé-liberté. Voir d'ailleurs, CE, 19 août 2002, Front national, Institut de formation des élus locaux : rec. CE 2002, p. 311 ; AJ 2992.1017, note X. Braud.

42. Jacques Fialaire et al., op. cit., p. 608.

43. Article premier de la DDHC: « Les hommes naissent et demeurent libres et égaux en droits. Les distinctions sociales ne peuvent être fondées que sur l'utilité commune. »

44. Jean-Nazaire Tama, Droit international et africain des droits de l'homme, Paris, L'Harmattan, 2012, p. 149. 
que ce droit bénéficie également à certains membres du corps social et en l'occurrence, les personnes ayant une orientation sexuelle différente de celle de la majorité, les opposants politiques ou encore les femmes victimes de discrimination au gré des vicissitudes de l'histoire.

L'importance du droit à la non-discrimination se mesure plus aisément à l'aune de la pratique qu'elle vise à juguler, à savoir la discrimination. Il faut toujours garder à l'esprit le fait que cette dernière, du point de vue juridique, vise à instaurer une «différenciation contraire au principe de l'égalité civile consistant à rompre celle-ci au détriment de certaines personnes physiques en raison de leur appartenance raciale ou confessionnelle, plus généralement par application de critères sur lesquels la loi interdit de fonder des distinctions juridiques arbitraires (sexe, opinions politiques, situation de famille, état de santé, handicap, origine, appartenance ou non-appartenance (vraie ou supposée) à une nation, une ethnie ou une race, activité syndicale ${ }^{45}$ )».

Dans les sociétés modernes, les groupes non dominants et en l'occurrence les peuples minoritaires doivent se voir appliquer le principe de non-discrimination qui est un rempart contre des fléaux tels que le racisme, la xénophobie ou l'intolérance religieuse. Cette exigence est aussi bien valable pour les États-nations que pour les États multinationaux. Les premiers se trouveront logiquement dans l'obligation d'accorder des droits identiques à l'ensemble de leurs populations s'ils ne veulent pas être en contradiction avec leur idéologie fondée sur l'unitarisme et le jacobinisme politico-juridique. Quant aux États multinationaux ou dont la nation se caractérise par une constitution hétérogène de la population qui forme quelquefois une mosaïque de peuples, le principe de non-discrimination permet d'éviter que des traitements juridiques différenciés ne soient source d'exclusion ou de frustration pour certaines communautés ethniques ou raciales.

C'est à juste titre que Norbert Rouland a pu dire qu'en consacrant le principe de non-discrimination, «les Constitutions africaines s'attachent à prévenir le danger particulariste sous la forme du tribalisme ${ }^{46}$ » ou de «l'ethnicisme». Mais au fond, une telle analyse est transposable, toute proportion gardée, à certains États d'Europe, d'Amérique ou d'Asie qui ont tout intérêt à concilier la diversité ethnique de la population, avec l'unité politique de l'État; d'où l'intérêt de déterminer avec précision la portée du droit à la non-discrimination.

45. Gérard Cornu, Vocabulaire juridique, Paris, PUF, 2011, p. 350, (voir «Discrimination»).

46. Norbert Rouland et al., op. cit., p. 287. 


\subsubsection{La portée du droit à la non-discrimination}

La portée du droit à la non-discrimination s'apprécie en réalité en fonction du texte juridique qui le consacre. Avant de pénétrer les droits nationaux, ce droit a d'abord été largement reconnu à l'échelle internationale notamment par la charte des Nations unies (art. $1 \S 3^{47}$ ), la Déclaration universelle des droits de l'homme (art. $2^{48}$ ), le PIDCP (art. $2 \$ 1^{49}$ ), la convention européenne des droits de l'homme (art. 14 ${ }^{50}$ ), la convention américaine relative aux droits de l'homme (art. $1 \$ 1^{51}$ ), la charte africaine des droits de l'homme et des peuples (art. $2^{52}$ ), la charte

47. Art. $1 \$ 3$ de la charte de l'ONU : «Les buts des Nations unies sont les suivants : “[...] 3- Réaliser la coopération internationale en résolvant les problèmes internationaux d'ordre économique, social, intellectuel ou humanitaire, en développant et en encourageant le respect des droits de l'homme et des libertés fondamentales pour tous, sans distinction de race, de sexe, de langue ou de religion." ”

48. Art. 2 de la déclaration universelle des droits de l'homme: «1. Chacun peut se prévaloir de tous les droits et de toutes les libertés proclamés dans la présente Déclaration, sans distinction aucune, notamment de race, de couleur, de sexe, de langue, de religion, d'opinion politique ou de toute autre opinion, d'origine nationale ou sociale, de fortune, de naissance ou de toute autre situation. 2. De plus, il ne sera fait aucune distinction fondée sur le statut politique, juridique ou international du pays ou du territoire dont une personne est ressortissante, que ce pays ou territoire soit indépendant, sous tutelle, non autonome ou soumis à une limitation quelconque de souveraineté.»

49. Art. $2 \S 1$ du PIDCP : «Les États parties au présent Pacte s'engagent à respecter et à garantir à tous les individus se trouvant sur leur territoire et relevant de leur compétence les droits reconnus dans le présent Pacte, sans distinction aucune, notamment de race, de couleur, de sexe, de langue, de religion, d'opinion politique ou de toute autre opinion, d'origine nationale ou sociale, de fortune, de naissance ou de toute autre situation.»

50. Art. 14 de la convention européenne des droits de l'homme: «La jouissance des droits et libertés reconnus dans la présente Convention doit être assurée, sans distinction aucune, fondée notamment sur le sexe, la race, la couleur, la langue, la religion, les opinions politiques ou toutes autres opinions, l'origine nationale ou sociale, l'appartenance à une minorité nationale, la fortune, la naissance ou toute autre situation.»

51. Art. $1 \$ 1$ de la convention américaine des droits de l'homme: "Les États parties s'engagent à respecter les droits et libertés reconnus dans la présente Convention et à en garantir le libre et plein exercice à toute personne relevant de leur compétence, sans aucune distinction fondée sur la race, la couleur, le sexe, la langue, la religion, les opinions politiques ou autres, l'origine nationale ou sociale, la situation économique, la naissance ou toute autre condition sociale.»

52. Art. 2 de la charte africaine des droits de l'homme et des peuples: « Toute personne a droit à la jouissance des droits et libertés reconnus et garantis dans la présente Charte sans distinction aucune, notamment de race, d'ethnie, de couleur, de sexe, de langue, de religion, d'opinion politique ou de toute autre opinion, d'origine nationale ou sociale, de fortune, de naissance ou de toute autre situation.» 
des droits fondamentaux de l'Union européenne (art. $21^{53}$ ) sans oublier la convention internationale sur l'élimination de toutes les formes de discrimination raciale ${ }^{54}$.

En droit régional (africain, américain et européen), il est admis que le droit à la non-discrimination soit en principe intrinsèquement lié aux autres droits garantis par le texte qui le prévoit. Toutefois, dans le cadre européen, par exemple, le juge de la Cour européenne des droits de l'homme a opté pour une application téléologique du principe de non-discrimination prévu par l'article 14 de la convention européenne des droits de l'homme dont les dispositions sont un complément aux autres clauses normatives de la convention ${ }^{55}$. L'interprétation du juge de la Cour européenne a permis finalement au principe de nondiscrimination de bénéficier d'une autonomie qui lui permet d'exister indépendamment des autres droits garantis par la convention européenne des droits de l'homme ${ }^{56}$; ce qui le rend plus en phase avec le régime juridique de la non-discrimination de l'article 26 du PIDCP.

En effet, avant même l'interprétation constructive de l'article $26 \mathrm{du}$ PIDCP par le Comité des droits de l'homme ${ }^{57}$, il est constant que ledit article apparaissait comme étant favorable à une application autonome du principe de non-discrimination puisqu'il dispose que «toutes les personnes sont égales devant la loi et ont droit sans discrimination à une égale protection de la loi». Un tel énoncé garantit donc un

53. Art. 21 de la charte des droits fondamentaux de l'Union européenne: «1. Est interdite, toute discrimination fondée notamment sur le sexe, la race, la couleur, les origines ethniques ou sociales, les caractéristiques génétiques, la langue, la religion ou les convictions, les opinions politiques ou toute autre opinion, l'appartenance à une minorité nationale, la fortune, la naissance, un handicap, l'âge ou l'orientation sexuelle. 2. Dans le domaine d'application du traité instituant la Communauté européenne et du traité sur l'Union européenne, et sans préjudice des dispositions particulières desdits traités, toute discrimination fondée sur la nationalité est interdite.»

54. La convention internationale sur l'élimination de toutes les formes de discrimination raciale a été adoptée et ouverte à signature par l'assemblée générale de l'ONU à travers sa résolution $2106 \mathrm{~A}(\mathrm{XX})$ du 21 décembre 1965. Elle est entrée en vigueur le 4 janvier 1969.

55. Voir notamment le raisonnement du juge dans l'affaire «relative à certains aspects du régime linguistique de l'enseignement en Belgique» (linguistique belge) du 23 juillet 1968 (Req. $\mathrm{n}^{\mathrm{o}} 1474 / 62 ; 1677 / 62 ; 1691 / 62 ; 1769 / 63 ; 1994 / 63 ; 2126 / 64$ ).

56. Voir à ce propos Frédéric Sudre, op. cit., p. 416-417.

57. Le Comité des droits de l'homme voit dans l'article $26 \mathrm{du}$ PIDCP une reconnaissance du principe général de l'égalité devant la loi qui émane notamment de l'article 7 de la déclaration universelle des droits de l'homme qui prohibe toute forme de discrimination dont l'État a pu se rendre coupable. Voir Déc. Broeks c/Pays-Bas, 9 avril 1987. 
principe d'égalité qui serait favorable à tous les individus placés sous la souveraineté juridique de l'État concerné. C'est une idée chère aux membres des peuples minoritaires qui peuvent ainsi bénéficier des droits individuels et collectifs reconnus aux autres citoyens; ce qui n'exclut d'ailleurs pas que des droits spécifiques puissent leur être accordés.

\section{Les droits spécifiques des peuples minoritaires contre la discrimination}

Après avoir considéré pendant longtemps que les minorités n'avaient pas besoin de se démarquer du reste de la population, on a tout de même fini par admettre qu'il était impérieux de leur reconnaître des droits particuliers. Il est donc utile de mettre en exergue le contenu de ces droits particuliers des peuples minoritaires (2.1) ainsi que les garanties de leur mise en œuvre (2.2).

\subsection{Le contenu des droits particuliers des peuples minoritaires}

Parmi les droits particuliers reconnus aux minorités, il faut évoquer le cas très important du droit à l'existence et à l'identité (2.1.1), mais aussi celui qui reconnaît aux minorités un traitement différencié qui leur est plutôt favorable; il s'agit, en d'autres termes, d'un droit à la discrimination positive (2.1.2).

\subsubsection{Le droit à l'existence et à l'identité des minorités}

Tout au long des siècles, la grande hantise des peuples minoritaires a été de voir leur existence compromise au sein d'États dans lesquels ils avaient du mal à être intégrés. Aussi, cette existence, avant tout physique, pouvait difficilement être dissociée de l'identité propre des peuples minoritaires ainsi concernés. C'est pourquoi, la déclaration des droits des personnes appartenant à des minorités nationales ou ethniques, religieuses et linguistiques prévoit dès son premier article $(\$ 1)$ que «les États protègent l'existence et l'identité nationale ou ethnique, culturelle, religieuse ou linguistique des minorités, sur leurs territoires respectifs, et favorisent l'instauration des conditions propres à promouvoir cette identité».

Toutefois, en droit international, la principale garantie de l'existence des peuples minoritaires réside dans la répression du génocide et du crime contre l'humanité. Le génocide, qui est sûrement le crime international le plus grave, est aujourd'hui considéré comme un acte visant à remettre en cause l'existence physique d'un groupe national, 
ethnique, racial ou religieux. Pourtant, Raphaël Lemkin, qui a développé le concept en $1944^{58}$, considérait que le génocide pouvait porter sur l'identité politique, culturelle, sociale ou même économique d'une minorité. D’ailleurs, il n'hésita pas à formuler des notions telles que le "génocide politique», le "génocide social », le "génocide culturel», le "génocide économique", "le génocide biologique», le "génocide religieux» ou encore le "génocide moral» pour qualifier, à côté du "génocide physique», un certain nombre d'actes criminels commis par les nazis ${ }^{59}$.

Aujourd'hui, on distingue clairement le génocide de l'ethnocide. Ce dernier est évoqué lorsqu'un groupe ethnique voit sa civilisation, sa culture et son tissu social détruits par des acteurs exogènes.

En 1948, la convention pour la prévention et la répression du crime de génocide a précisé que ce crime «s'entend de l'un quelconque des actes [...], commis dans l'intention de détruire, en tout ou en partie, un groupe national, ethnique, racial ou religieux, comme tel : (a) Meurtre de membres du groupe ; (b) Atteinte grave à l'intégrité physique ou mentale de membres du groupe; (c) Soumission intentionnelle du groupe à des conditions d'existence devant entraîner sa destruction physique totale ou partielle ; (d) Mesures visant à entraver les naissances au sein du groupe ; (e) Transfert forcé d'enfants du groupe à un autre groupe ».

Le critère déterminant dans la définition du génocide qui est «l'intention de détruire en tout ou en partie, un groupe national, racial, ethnique ou religieux» a été repris par les différents statuts des juridictions pénales internationales ${ }^{60}$. Dans la résolution 96 (I) du 11 décembre 1946, l'assemblée générale des Nations unies affirme que le génocide est «le refus du droit à l'existence de groupes entiers, de même que l'homicide est le refus du droit à l'existence d'un individu». Quant aux crimes contre l'humanité, le fait qu'ils soient liés à « une attaque généralisée ou systématique lancée contre toute population civile ${ }^{61}$ » peut faire d'eux des actes visant à remettre en cause l'existence physique des membres d'un peuple minoritaire.

58. Avant la fin de la seconde guerre mondiale, Raphaël Lemkin proposa en 1944 la notion de génocide dans l'ouvrage, Axis Rule in Occupied Europe (Washington, Carnegie Endowment for World Peace, 1944).

59. Sur la référence aux différentes formes de génocide évoquées par Raphaël Lemkin, lire Gaetano Pentassuglia, op. cit., p. 83.

60. Voir l'article $4 \$ 2$ du statut du Tribunal pénal international pour l'ex-Yougoslavie, l'article $2 \S 2$ du statut du Tribunal pénal international pour le Rwanda et l'article 6 du statut de la Cour pénale internationale.

61. Voir l'article $7 \mathrm{du}$ statut de la Cour pénale internationale. 
Dans le cadre européen, l'intégrité physique des membres des peuples minoritaires a même pu être incorporée dans le domaine de l'article 3 de la convention européenne des droits de l'homme qui porte sur l'interdiction de la torture. Ainsi la Cour européenne des droits de l'homme a considéré que l'invocation de l'article 3 susévoqué peut résulter de l'expulsion forcée d'un étranger appartenant «à un groupe minoritaire particulièrement menacé ${ }^{62}$ ».

En plus de ne pas porter atteinte à leur intégrité physique, les États qui abritent les minorités ne doivent pas fouler aux pieds l'identité de ces dernières. Ils doivent notamment s'abstenir de mener toute politique visant à forcer des peuples minoritaires à abandonner ce qui fait leur spécificité sur le plan culturel, linguistique ou religieux. Au niveau européen, l'article $5(\$ 1)$ de la convention-cadre pour la protection des minorités nationales prévoit que «les Parties s'engagent à promouvoir les conditions propres à permettre aux personnes appartenant à des minorités nationales de conserver et développer leur culture, ainsi que de préserver les éléments essentiels de leur identité que sont leur religion, leur langue ${ }^{63}$, leurs traditions et leur patrimoine culturel». Aussi, la déclaration des Nations unies sur les droits des personnes appartenant à des minorités nationales ou ethniques, religieuses et linguistiques (art. $2 \$ 1$ ), insiste sur le droit de ceux-ci de «jouir de leur propre culture, de professer et de pratiquer leur propre religion, et d'utiliser leur propre langue en privé et en public, librement et sans ingérence ni discrimination quelconque».

Outre un droit à l'existence et à l'identité, la vulnérabilité des minorités a poussé certains États à les faire bénéficier de «mesures spéciales ${ }^{64}$ » sous la forme d'un droit à la discrimination positive.

\subsubsection{Le droit des minorités à la discrimination positive}

La situation particulière des minorités peut justifier que leur soit accordé un traitement différencié ou préférentiel par rapport aux autres membres de la société sans que les mesures prises en leur faveur ne

62. Lire notamment l'affaire Salah Sheekh c/Pays-Bas (11 janvier 2007). Voir aussi Frédéric Sudre, op. cit., p. 99.

63. Voir aussi la charte européenne des langues régionales ou minoritaires adoptée par le Comité des ministres du Conseil de l'Europe le 25 juin 1992.

64. Formule utilisée par l'article $1^{\mathrm{er}} \$ 4$ de la convention internationale sur l'élimination de toutes les formes de discrimination raciale, adoptée et ouverte à la signature et à la ratification par l'assemblée générale de l'ONU dans sa résolution $2106 \mathrm{~A}(\mathrm{XX}) \mathrm{du}$ 21 décembre 1965. 
constituent une entorse au sacro-saint principe d'égalité ${ }^{65}$. C'est en tout cas l'objectif visé par la discrimination positive qui est de mise dans un certain nombre d'États. Avant même ses premiers balbutiements dans la société américaine au cours des années $1960^{66}$, le droit à la discrimination positive ("positive discrimination", "affirmative action", "positive action») a bénéficié d'une certaine consécration sur le plan international grâce notamment à la CPJI avec la décision concernant les écoles minoritaires en Albanie rendue le 6 avril 1935.

La discrimination positive est un ensemble de traitements différenciés ou préférentiels qui visent à réduire les inégalités socio-économiques dont sont victimes certains groupes humains par rapport au reste de la population. Il est vrai que sa pratique peut varier selon les États où elle est mise en œuvre. Ainsi, aux États-Unis, par exemple, elle « désigne l'ensemble des mesures qui octroient aux membres de groupes ayant été soumis dans le passé à un régime juridique discriminatoire, un traitement préférentiel dans la répartition de certaines ressources ${ }^{67}$ ». Mais, il faut préciser que la discrimination positive ne concerne pas que les groupes minoritaires, puisqu'elle bénéficie très souvent aux catégories non dominantes telles que les femmes qui peuvent ainsi rattraper le retard socio-économique ou même politique ${ }^{68}$ lié au traitement inégalitaire dont elles ont été victimes au fil des siècles.

65. L'article $1^{\text {er }} \$ 4$ de la convention internationale sur l'élimination de toutes les formes de discrimination raciale prévoit que «les mesures spéciales prises à seule fin d'assurer comme il convient le progrès de certains groupes raciaux ou ethniques ou d'individus ayant besoin de la protection qui peut être nécessaire pour leur garantir la jouissance et l'exercice des droits de l'homme et des libertés fondamentales dans des conditions d'égalité ne sont pas considérées comme des mesures de discrimination raciale, à condition toutefois qu'elles n'aient pas pour effet le maintien de droits distincts pour des groupes raciaux différents et qu'elles ne soient pas maintenues en vigueur une fois atteints les objectifs auxquels elles répondaient ».

66. Aux États-Unis, c'est dans la foulée des revendications en faveur des droits civiques des minorités que le Civil Rights Act va être adopté (1964). Le titre VII de ce texte va être considéré par les juridictions américaines comme le fondement du droit pour les minorités de bénéficier de la discrimination positive (affirmative action). Sur la question, lire notamment Daniel Sabbagh, L'égalité par le droit. Les paradoxes de la discrimination positive aux États-Unis, Paris, Economica, 2003, p. 59 et suiv.; Éric Keslassy, De la discrimination positive, Levallois-Perret, Bréal, 2004, spéc. p. 11.

67. Daniel Sabbagh, «La tentation de l'opacité: le juge américain et l'affirmative action dans l'enseignement supérieur", Pouvoirs, $\mathrm{n}^{\circ} 111$, novembre 2004, p. 5.

68. En France, par exemple, la loi du 6 juin 2000 sur l'égal accès des femmes et des hommes aux mandats électoraux et fonctions électives, instaure une discrimination positive favorable aux femmes en ce qui concerne l'accès à la vie politique. 
Les raisons de la discrimination positive en faveur des minorités ont été clairement exposées par la CPJI dans la décision concernant les écoles minoritaires en Albanie précitée. Cette juridiction a en effet été pragmatique dans son raisonnement en considérant que «l'égalité en droit exclut toute discrimination: l'égalité en fait peut, en revanche, rendre nécessaires des traitements différents en vue d'arriver à un résultat qui établisse l'équilibre entre des situations différentes [...]». «L'égalité entre majoritaires et minoritaires doit être une égalité effective, réelle ${ }^{69}$.» C'est au nom d'une égalité réelle favorable aux minorités que la déclaration des droits des personnes appartenant à des minorités nationales ou ethniques, religieuses et linguistiques recommande aux États de prendre toutes les mesures permettant aux membres de leurs peuples minoritaires «d'exercer intégralement et effectivement tous les droits de l'homme et toutes les libertés fondamentales ${ }^{70} »$. Aussi, dans le cadre européen, le préambule du protocole $\mathrm{n}^{\mathrm{O}} 12$ de la convention de sauvegarde des droits de l'homme et des libertés fondamentales (4 novembre 2000) admet que «le principe de non-discrimination n'empêche pas les États parties de prendre des mesures afin de promouvoir une égalité pleine et effective, à la condition qu'elles répondent à une justification objective et raisonnable».

La discrimination positive instaurée par un État doit avoir un caractère raisonnable et objectif, et le fait qu'elle soit mise en ouvre au profit d'un groupe minoritaire particulier ne doit pas avoir pour effet de créer une discrimination à l'égard d'autres populations minoritaires qui pourraient objectivement bénéficier elles aussi d'un traitement différencié. C’est en tout cas le point de vue exprimé par le Comité des droits de l'homme de l'ONU dans l'affaire Waldman $c /$ Canada $^{71}$. La décision de ce pays d'accorder des privilèges aux écoles catholiques romaines de l'Ontario à l'exclusion de celles des autres écoles religieuses minoritaires $^{72}$ fut considérée par le Comité des droits de l'homme comme

69. CPJI, Écoles minoritaires en Albanie, 6 avril 1935, série A/B, nº 64, p. 19. Dans l'avis consultatif du 10 septembre 1923 au sujet de l'affaire des colons allemands en Pologne (avis $\mathrm{n}^{\mathrm{o}} 6$ ), la CPJI affirme déjà qu' « il faut qu'il y ait égalité de fait et non seulement égalité formelle en droit en ce sens que les termes de la loi évitent d'établir un traitement différentiel».

70. Lire l'article $4 \$ 1$ de la déclaration des droits des personnes appartenant à des minorités nationales ou ethniques, religieuses et linguistiques, (Assemblée générale de l'ONU, rés. 47/135, 18 déc. 1992).

71. Comité des droits de l'homme, affaire Waldman c/Canada, communication $n^{0}$ 694/1996, observations du 3 novembre 1999, CCPR/C/67/D/694/1996.

72. Sur le sujet, lire Gaetano Pentassuglia, op. cit., p. 113. 
contraire au principe d'égalité prévu dans l'article $26 \mathrm{du} \mathrm{PIDCP}^{73}$. C'est sans doute pour être conforme à cette exigence que la discrimination positive américaine (affirmative action) concerne aussi bien la minorité noire, que les minorités hispanique, asiatique et amérindienne qui bénéficient toutes de «mesures spéciales» en ce qui concerne l'accès à l'université, à l'emploi ou à la passation des marchés publics ${ }^{74}$.

Il est vrai que la discrimination positive est mise en œuvre dans des pays ayant connu des trajectoires historiques différentes. Les inégalités qu'elle vise à corriger peuvent provenir soit d'une soumission économique, culturelle ou politique organisée par un groupe dominant au détriment des groupes dominés, c'est le cas dans des pays comme l'Inde, les États-Unis, ou l'Afrique du Sud ${ }^{75}$, soit du morcellement géographique d'entités politiques ayant placé certaines populations dans un statut de minorités vulnérables comme c'est le cas dans certains pays d'Europe centrale ou orientale ${ }^{76}$.

Il faut souligner que, même en France, l'article 24 de la loi organique no 99-209 du 19 mars 1999 relative à la Nouvelle-Calédonie, consécutive à l'accord de Nouméa ${ }^{77}$, consacre, en Nouvelle-Calédonie, une discrimination positive dans le domaine de l'emploi au profit des Néocalédoniens ${ }^{78}$.

73. Art. 26 du PIDCP : «Toutes les personnes sont égales devant la loi et ont droit sans discrimination à une égale protection de la loi. À cet égard, la loi doit interdire toute discrimination et garantir à toutes les personnes une protection égale et efficace contre toute discrimination, notamment de race, de couleur, de sexe, de langue, de religion, d'opinion politique et de toute autre opinion, d'origine nationale ou sociale, de fortune, de naissance ou de toute autre situation.»

74. Lire Éric Keslassy, op. cit., p. 11.

75. Voir Laure Bereni et al., op. cit., p. 29. Il faut tout de même préciser qu'en ce qui concerne l'Afrique du Sud, les inégalités sociales sont en grande partie liées à la politique d'apartheid mise sur pied pendant des décennies (de 1948 à 1991) par la minorité blanche au détriment principalement de la majorité noire.

76. Néanmoins, en France, «l'universalisme républicain» qui n’est pas favorable à la reconnaissance de l'existence des minorités a conduit les pouvoirs publics à développer le concept de discrimination positive territoriale au profit des espaces urbains défavorisés. Sur le sujet lire Milena Doytcheva, Une discrimination positive à la française? Ethnicité et territoire dans les politiques de la ville, Paris, La Découverte, 2007, $226 \mathrm{p}$.

77. Voir l'accord sur la Nouvelle-Calédonie signé à Nouméa le 5 mai 1998.

78. Art. 24 de la loi organique $n^{0}$ 99-209 du 19 mars 1999 relative à la NouvelleCalédonie : «Dans le but de soutenir ou de promouvoir l'emploi local, la NouvelleCalédonie prend au bénéfice des citoyens de la Nouvelle-Calédonie et des personnes qui justifient d'une durée suffisante de résidence des mesures visant à favoriser l'exercice d'un emploi salarié, sous réserve qu'elles ne portent pas atteinte aux avantages individuels et collectifs dont bénéficient à la date de leur publication les 
Toutefois, nonobstant la divergence des situations dans les États concernés, le droit international considère que la discrimination positive doit se manifester dans des actions "spéciales et concrètes ${ }^{79}$ ", même si globalement, un certain nombre de garanties conditionnent la mise en œuvre des droits particuliers en faveur des minorités.

\subsection{Les garanties pour la mise en œuvre des droits particuliers des peuples minoritaires}

Pour que les droits particuliers des peuples minoritaires puissent être mis en œuvre, certaines garanties doivent être apportées à la fois par les États (2.2.1) et par les minorités elles-mêmes (2.2.2).

\subsubsection{Les garanties apportées par les États}

Les États doivent en principe donner des gages de leur engagement à préserver les droits des minorités. Ces gages se traduisent en général par la mise en œuvre d'institutions démocratiques seules à même de protéger les peuples minoritaires contre la discrimination. L'adaptation du droit national par rapport aux exigences du droit international des minorités passe par l'insertion de certaines règles spécifiques du droit international des droits de l'homme dans l'ordre juridique interne, ce qui peut avoir comme conséquence, une modification de la Constitution ou plus largement de la législation nationale. Les États peuvent aussi être amenés à créer des services techniques chargés de protéger les minorités contre toute discrimination de jure ou de facto. C'est en tout cas ce que

autres salariés. De telles mesures sont appliquées dans les mêmes conditions à la fonction publique de la Nouvelle-Calédonie et à la fonction publique communale. La Nouvelle-Calédonie peut également prendre des mesures visant à restreindre l'accession à l'exercice d'une profession libérale à des personnes qui ne justifient pas d'une durée suffisante de résidence. La durée et les modalités de ces mesures sont définies par des lois du pays.»

79. Voir notamment l'article $2 \S 2$ de la convention internationale sur l'élimination de toutes les formes de discrimination raciale du 21 décembre 1965 qui prévoit que " [1] es États parties prendront, si les circonstances l'exigent, dans les domaines social, économique, culturel et autres, des mesures spéciales et concrètes pour assurer comme il convient le développement ou la protection de certains groupes raciaux ou d'individus appartenant à ces groupes en vue de leur garantir, dans des conditions d'égalité, le plein exercice des droits de l'homme et des libertés fondamentales ». "Ces mesures ne pourront en aucun cas avoir pour effet le maintien de droits inégaux ou distincts pour les divers groupes raciaux, une fois atteints les objectifs auxquels elles répondaient. » 
préconise, au sein du Conseil de l'Europe, l'article $4 \$ 2$ de la conventioncadre pour la protection des minorités nationales qui dispose que «les Parties s'engagent à adopter, s'il y a lieu, des mesures adéquates en vue de promouvoir, dans tous les domaines de la vie économique, sociale, politique et culturelle, une égalité pleine et effective entre les personnes appartenant à une minorité nationale et celles appartenant à la majorité».

C'est à l'occasion des guerres civiles ou des tensions politiques menaçant la sécurité des groupes non dominants que les États concernés se trouvent parfois contraints d'apporter des garanties supplémentaires à la protection des minorités qu'ils abritent. Ainsi en 1991, fut adopté en exYougoslavie un document dans le cadre d'une conférence internationale connu sous le nom de "Disposition du traité pour la Convention" ou «Projet Carrington». Gaetano Pentassuglia fait remarquer que ce projet engageait les entités politiques concernées à protéger les minorités nationales ou ethniques comme l'exigeaient les normes de l'ONU, de la CSCE et du Conseil de l'Europe ${ }^{80}$. Par ailleurs, dans un contexte de vives tensions politiques, la Commission d'arbitrage de la conférence européenne pour la paix en Yougoslavie ${ }^{81}$ précise dans un avis rendu le 11 janvier 1992 qu' "en vertu de normes, maintenant impératives du droit international général, il appartient aux États d'assurer le respect des droits des minorités ». « Cette exigence s'impose à toutes les Républiques à l'égard des minorités établies sur leur territoire ${ }^{82}$.»

Le 14 décembre 1995, pour mettre un terme aux affrontements interethniques en Bosnie-Herzégovine, les accords de Dayton (signé par les autorités de Bosnie-Herzégovine, de Croatie et de la République fédérale de Yougoslavie) n'oublient pas de mentionner des obligations visant à garantir les droits des minorités (annexe $4^{83}$ ) et plus globalement les droits de l'homme (annexe 6).

Dans le cadre de la crise en Irlande du Nord, un accord de paix conclu le 10 avril 1998 entre le gouvernement du Royaume-Uni et le gouvernement d'Irlande contient en filigrane des dispositions visant

80. Gaetano Pentassuglia, op. cit., p. 255.

81. Sur le rôle de cette commission lire Alain Pellet, "L'activité de la Commission d'arbitrage de la Conférence européenne pour la paix en Yougoslavie ", AFDI 1992, $\mathrm{n}^{\mathrm{o}} 38$, p. 220-238.

82. Voir Commission d'arbitrage de la conférence européenne pour la paix en Yougoslavie, Populations serbes de Croatie et Bosnie-Herzégovine, avis $\mathrm{n}^{0} 2, \$ 2,11$ janvier 1992.

83. Voir plus précisément l'article II-4 de la Constitution de Bosnie-Herzégovine contenue dans l'annexe 4 des accords de Dayton. 
à préserver les droits des catholiques, qui constituent une minorité dans l'ensemble du Royaume-Uni et en Irlande du Nord, mais aussi ceux des protestants qui deviendraient un groupe minoritaire dans une Irlande unifiée ${ }^{84}$. Il ressort de cet accord qui reconnaît le droit à l'autodétermination des populations concernées que «quel que soit le choix librement opéré par la majorité du peuple d'Irlande du Nord, les pouvoirs du gouvernement souverain compétent en Irlande du Nord seront exercés dans une stricte impartialité au nom de l'ensemble du peuple, compte tenu de la diversité de ses identités et traditions, et reposeront sur les principes du plein respect et de l'égalité des droits civils, politiques, sociaux et culturels, de la non-discrimination pour tous les citoyens, et d'une même estime et d'un traitement juste et égal des identités, génies et aspirations respectifs des deux communautés ${ }^{85}$ ».

La pratique juridique internationale conduit donc généralement les États à apporter un florilège de garanties pour la protection des droits des peuples minoritaires de manière à les préserver contre les discriminations. Toutefois, ces minorités sont elles aussi soumises à des exigences qui apparaissent comme autant de garanties qu'elles doivent apporter aux États qui les abritent.

\subsubsection{Les garanties apportées par les minorités}

Les États n'ont pas que des obligations à l'égard des peuples minoritaires qu'ils doivent protéger contre la discrimination; ils sont aussi en droit d'exiger que ceux-ci respectent les institutions et les principes démocratiques mis en œuvre par la majorité. De plus, le fait qu'un groupe constitue une minorité sur l'ensemble territorial d'un État n'exclut pas qu'il soit en fait majoritaire dans une région ou dans une localité bien précise dudit État. Dès lors, ce groupe devrait éviter tout comportement qui aurait pour effet de compromettre le bénéfice de leurs droits par les membres des autres communautés qui constituent l'État.

Par ailleurs, le fait qu'une minorité sur le plan national constitue en fait une majorité sur une portion d'un territoire étatique peut faire courir le risque que ladite minorité développe un sentiment nationaliste qui aurait pour effet d'accroître les velléités sécessionnistes pouvant faire voler en éclats l'intégrité territoriale de certains États. Pour éviter une telle situation, le droit international a consacré le principe de l'intangibilité

84. Voir l'analyse de Gaetano Pentassuglia, op. cit., p. 258.

85. Voir le $\$ 5$ (v) de l'accord conclu en Irlande du Nord entre le Royaume-Uni de Grande-Bretagne et d'Irlande du Nord et l'Irlande (10 avril 1998). 
des frontières avec la règle uti possidetis juris. Néanmoins, il n'est pas du tout aisé d'établir l'illicéité d'une sécession sur le plan international. Il est d'ailleurs admis que, "quelle que soit sa légalité au plan interne, la sécession est un fait politique au regard du droit international, qui se contente d'en tirer les conséquences lorsqu'elle aboutit à la mise en place d'autorités étatiques effectives et stables ${ }^{86} "$.

Si dans l'absolu le droit à la sécession n'a été reconnu expressément qu'aux peuples coloniaux ${ }^{87}$, l'histoire regorge d'exemples d'entités non coloniales nées suite à leur détachement d'un État qui a ainsi vu son intégrité territoriale remise en cause notamment par des peuples minoritaires. Bon nombre de ces nouvelles entités politiques créées par des minorités ont d'ailleurs réussi à accéder officiellement à la souveraineté internationale après une reconnaissance de jure ou de facto $^{88}$. Ainsi, les populations juives en Palestine, constituant au départ un peuple minoritaire organisé au sein des kibboutzim dès les années 1910, ont pu, suite à la Shoah, proclamer l'indépendance de l'État d'Israël le 14 mai 1948. Le nouvel État fut reconnu de facto par les États-Unis moins d'une douzaine de jours après sa création. De même, entre 1967 et 1969, la France soutint l'éphémère république du Biafra ${ }^{89}$ créée par l'ethnie Ibo majoritaire dans le Biafra (70 \% de la population), mais minoritaire dans tout le Nigéria ( $18 \%$ de la population) qui évita in extremis une sécession ${ }^{90}$.

Un autre cas emblématique de sécession sous l'instigation d'un peuple minoritaire est l'indépendance du Kosovo déclarée le 17 février 2008. En effet, bien qu'étant une minorité dans l'ensemble de la République fédérale de Yougoslavie (RFY), les Albanais majoritaires dans la région du Kosovo ont pu créer un nouvel État, alors même que, ni l'accord de Rambouillet (accord intérimaire pour la paix et l'autonomie au Kosovo) du 27 mai 1999 ni la résolution 1244 (1999) du Conseil de sécurité de l'ONU, instaurant une administration internationale

86. Patrick Daillier, Mathias Forteau et Alain Pellet, Droit international public, Paris, LGDJ-Lextenso, 2009, p. 585.

87. Sur le sujet lire Jean Combacau et Serge Sur, Droit international public, Paris, Lextenso, 2014, p. 272.

88. Sur la reconnaissance de jure (définitive avec tous les effets de la reconnaissance) ou de facto (provisoire et révocable) des États, lire Pierre-Marie Dupuy et Yann Kerbrat, Droit international public, Paris, Dalloz, 2014, p. 38.

89. Après une guerre civile particulièrement meurtrière (environ 1 million de morts), le Biafra réintégra le Nigéria le 15 janvier 1970.

90. Lire Patrick Daillier, Mathias Forteau et Alain Pellet, op. cit., p. 586. 
civile (MINUK) n'avaient admis la possibilité que soit remise en cause l'intégrité territoriale de la RFY.

Après le soutien de l'indépendance du Kosovo par les chancelleries occidentales, celle-ci a pu être homologuée le 22 juillet 2010 par la CIJ qui a reconnu la conformité au droit international de l'indépendance unilatérale du Kosovo ${ }^{91}$ malgré les critiques acerbes de la Russie. Par ailleurs, le 9 juillet 2011, la minorité chrétienne du Soudan a proclamé l'indépendance du sud Soudan au grand dam de Khartoum. Ce nouvel État a ensuite très rapidement été admis au sein de l'assemblée générale des Nations unies, dès le 14 juillet, soit seulement cinq jours après sa sécession.

Il faut en outre préciser que la Russie, qui s'est vertement opposée à l'indépendance du Kosovo en février 2008, a soutenu militairement au mois d'août de la même année l'indépendance des républiques géorgiennes d'Abkhazie et d'Ossétie du Sud ${ }^{92}$. Qui plus est, en mars 2014, la minorité russophone de la république autonome de Crimée a pu organiser une sécession du reste de l'Ukraine avec le soutien politique et la pression militaire de Moscou $^{93}$.

Finalement, force est de constater que si les peuples minoritaires ont en principe une obligation de loyauté vis-à-vis des États où ils sont établis, il n'en demeure pas moins que rien ne garantit en réalité que celle-ci sera respectée. Ce faisant, si la sécession d'un territoire organisée par une minorité peut apparaître de prime abord comme une violation du droit national et plus précisément du droit constitutionnel, le droit international la considère en général comme une simple situation de fait $^{94}$, à moins qu'elle ne soit, par exemple, liée à un acte d'agression dont l'illicéité ne fait aucun doute sur le plan international.

91. Voir CIJ, avis consultatif du 22 juillet 2010, «Conformité au droit international de la déclaration unilatérale d'indépendance relative au Kosovo », dans Recueil des arrêts, avis consultatifs et ordonnances (2010), disponible sur le site http://www.icj-cij.org.

92. Sur le sujet, lire Raphaële Rivier, Droit international public, Paris, PUF, 2013, p. 285.

93. Le parlement de Crimée a proclamé l'indépendance de son entité politique par rapport à l'Ukraine le 11 mars 2014 et le 16 mars les habitants de la Crimée se sont prononcés en faveur d'un rattachement de leur territoire à la Russie. Deux jours plus tard (le 18 mars), c'est le président russe Vladimir Poutine qui signe avec les autorités de la Crimée l'incorporation de cette péninsule à la Russie. Ladite incorporation a été ratifiée (443 voix pour et 1 seule contre) par la chambre basse du parlement russe (la Douma) le 20 mars 2014.

94. Raphaële Rivier a pu constater qu' "aujourd'hui comme autrefois des sécessions s'accomplissent hors décolonisation au bénéfice du contexte politique». "Les facteurs juridiques n'y ont qu'un poids subsidiaire» (op. cit., p. 284). Lire aussi Théodore Christakis, «La sécession : une question de simple fait? », septembre 2007, $15 \mathrm{p}$. 
L'histoire a démontré que les vicissitudes qui ont accompagné le processus de construction des États modernes ont très souvent placé les peuples minoritaires dans une situation inconfortable, soit parce qu'ils faisaient l'objet d'une assimilation forcée au sein des pays qui les abritaient, soit parce qu'ils étaient victimes d'une marginalisation ou même d'une tentative d'extermination par les entités politiques qui les rejetaient. Il est vrai que, depuis quelques décennies le droit international a permis aux peuples minoritaires de bénéficier d'un certain nombre de règles juridiques qui sont censées les protéger contre les discriminations. Néanmoins, il n'existe pas au niveau des États, un régime juridique uniforme visant à préserver les groupes non dominants contre la majorité qui peut être tentée d'abuser des pouvoirs qui découlent des effets du système de démocratie politique qui lui est favorable.

Dans les États-nations comme la France, l'édiction des normes juridiques est tributaire du principe du jacobinisme étatique et des idéaux de la philosophie libérale favorables à une simple reconnaissance des droits individuels à l'ensemble des citoyens. Les États multinationaux, à l'instar du Canada, seront globalement beaucoup plus sensibles à une reconnaissance des droits particuliers en faveur des minorités qui peuvent même bénéficier d'un traitement différencié comme le suggère le droit international, afin qu'elles puissent défendre leur identité culturelle ou linguistique.

Il faut dire que si l'ordre juridique international laisse une grande marge de manœuvre aux États en ce qui concerne la manière dont ils devraient garantir les droits des minorités, il n'en demeure pas moins que, dans tous les pays démocratiques, celles-ci bénéficient d'une manière générale du sacro-saint principe de non-discrimination. Toutefois, la manière dont le droit international applicable aux minorités sera mis en ouvre en droit interne dépendra la plupart du temps de la situation politique des États concernés. Ainsi, en cas de développement d'un repli identitaire chez des minorités pouvant déboucher par exemple sur des velléités sécessionnistes dans une partie de leur territoire, les États n'hésiteront pas à prendre des mesures exceptionnelles pour protéger leur intégrité territoriale; ce qui ne se fera pas sans incidence négative sur les droits fondamentaux des peuples minoritaires.

Docteur en Droit public Enseignant vacataire à l'École de droit de l'université d'Auvergne 